\title{
LABORATORY ANGULAR DISTRIBUTIONS FOR THE PRODUCTION OF CHARGED SECONDARIES IN INELASTIC PROTON-PROTON COLLISIONS AT $102 \mathrm{GeV} / c *$
}

\author{
J. COOPER, A. SEIDL and J.C. VANDER VELDE \\ University of Michigan, Ann Arbor, Michigan 48104 \\ C. BROMBERG, D. COHEN **, J.P. DE BRION ***, \\ T. FERBEL and P. SLATTERY \\ University of Rochester, Rochester, New York 14627
}

Received 18 June 1974

\begin{abstract}
Lab angular distributions for the production of charged secondary particles in inelastic proton-proton collisions are presented. Data are separately displayed for protons and for positive and negative mesons (pions and kaons combined).
\end{abstract}

\section{Introduction}

One motivation underlying the initial set of bubble chamber exposures at NAL was to obtain reliable information concerning the general characteristics of particle collisions in this heretofore unexplored high-energy range in order that future higher statistics experiments could be optimally planned. With this goal in mind, we here present lab angular distributions for the production of charged secondary particles in proton-proton collisions at $102 \mathrm{GeV} / c$. Such distributions we believe represent the most convenient and immediately accessible summary of the type of survey information which is most useful to the planning of the widest variety of experiments.

The data are from a 30000 frame exposure of the NAL/ANL 30 inch liquid hydrogen bubble chamber to a beam of $102 \mathrm{GeV} / c$ protons. Approximately 2500 events were measured and spatially reconstructed using a fiducial volume which provided at least $20 \mathrm{~cm}$ of path length for each outgoing track. The full exposure was scanned and measured for events in the 2 to 8 pronged topologies, while approximately one

* Research supported by the US Atomic Energy Commission. Computing funds at the University of Rochester are provided by the University.

** Present address: Nevis Laboratories, Columbia University.

*** Permanent address: CEN Saclay, France. 

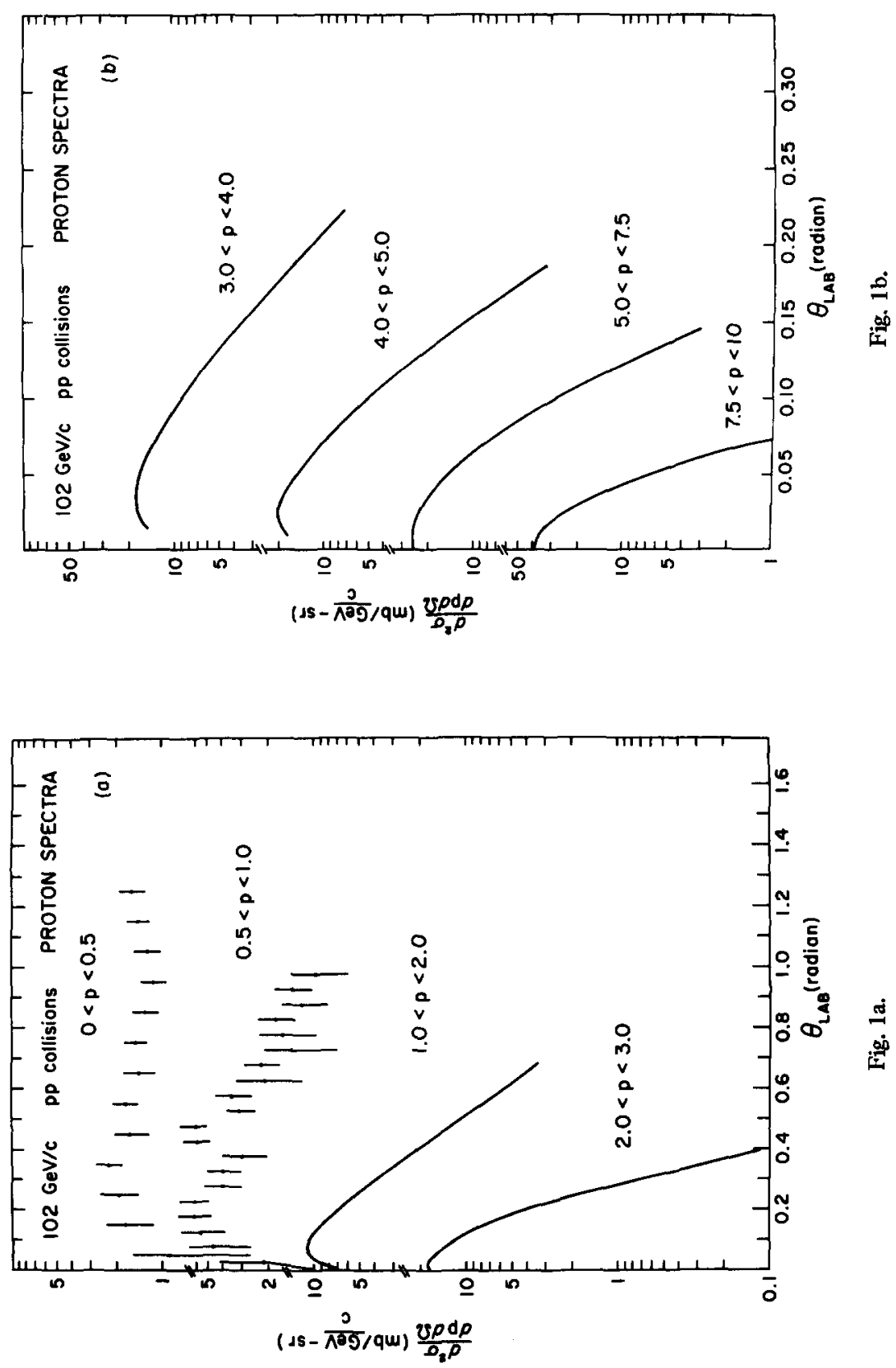

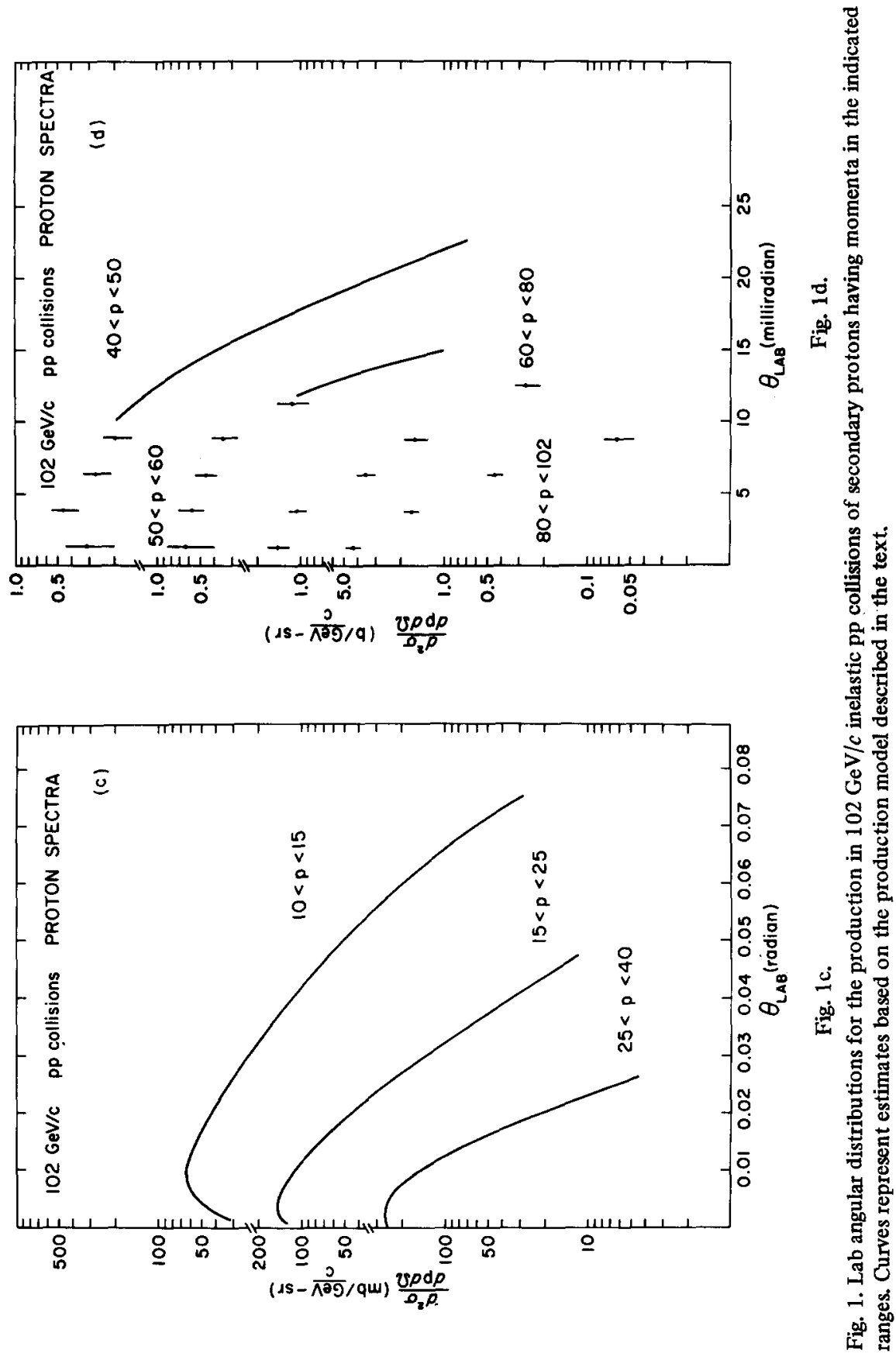

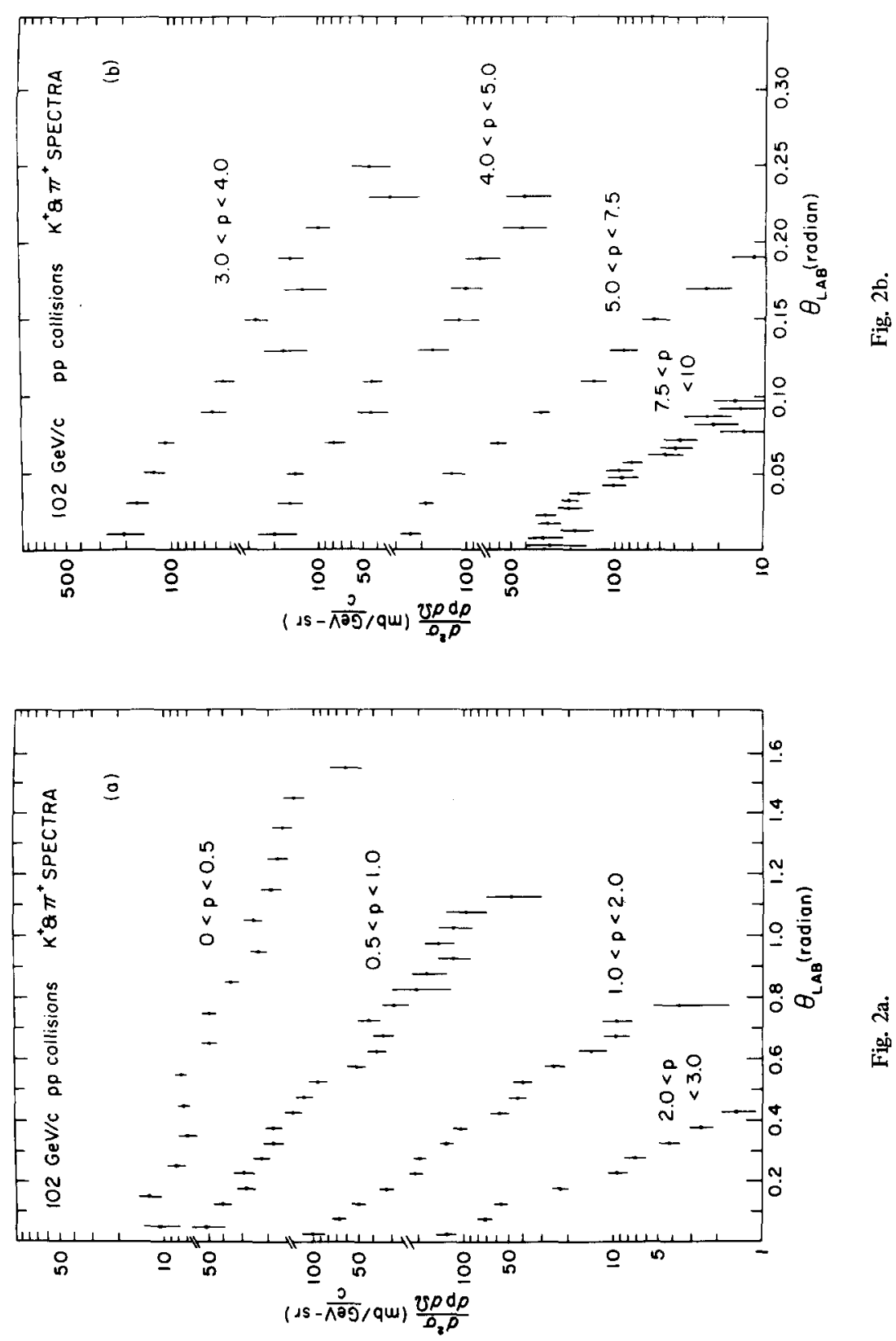


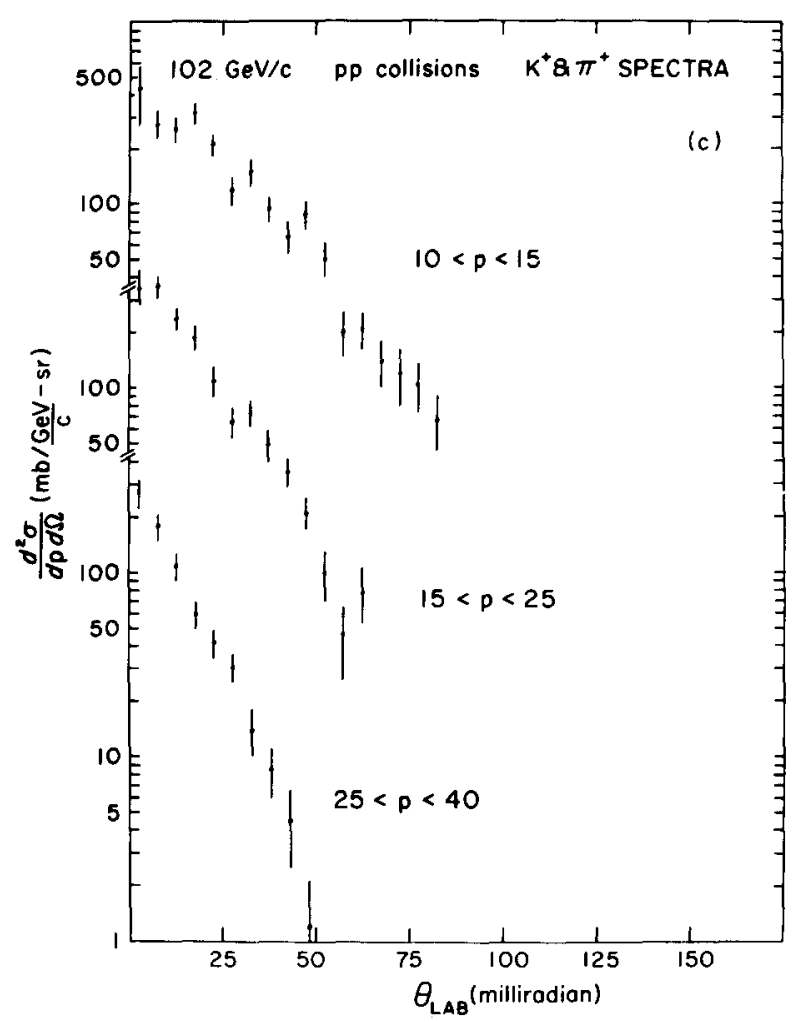

Fig. 2c.

Fig. 2. Lab angular distributions for the production in $102 \mathrm{GeV} / \mathrm{c}$ inelastic pp collisions of positively charged pions and kaons having momenta in the indicated ranges. Protons have been removed using the production model described in the text.

half the film was analyzed to obtain the sample of events from the higher topologies. The resulting contribution of each topology to the overall data set was individually normalized using topological cross sections which were obtained from a scan of the entire exposure [1]. Measuring and reconstruction losses arising from severe overlap between outgoing tracks or from secondary interactions very near the production vertex accounted for less than $5 \%$ of the detected events in any particular topology. These losses were assumed to be unbiased for the purposes of this paper.

A kinematic fitting procedure was employed to identify elastic scattering events [2]. These events were subsequently removed from the two prong sample, and consequently the angular distributions to be presented are for inelastic events only. To improve the overall resolution, only tracks which were backward in the overall c.m. were used in the analysis. The symmetry of the proton-proton interaction was then invoked to obtain the sample of forward going tracks. The error bars shown include, 

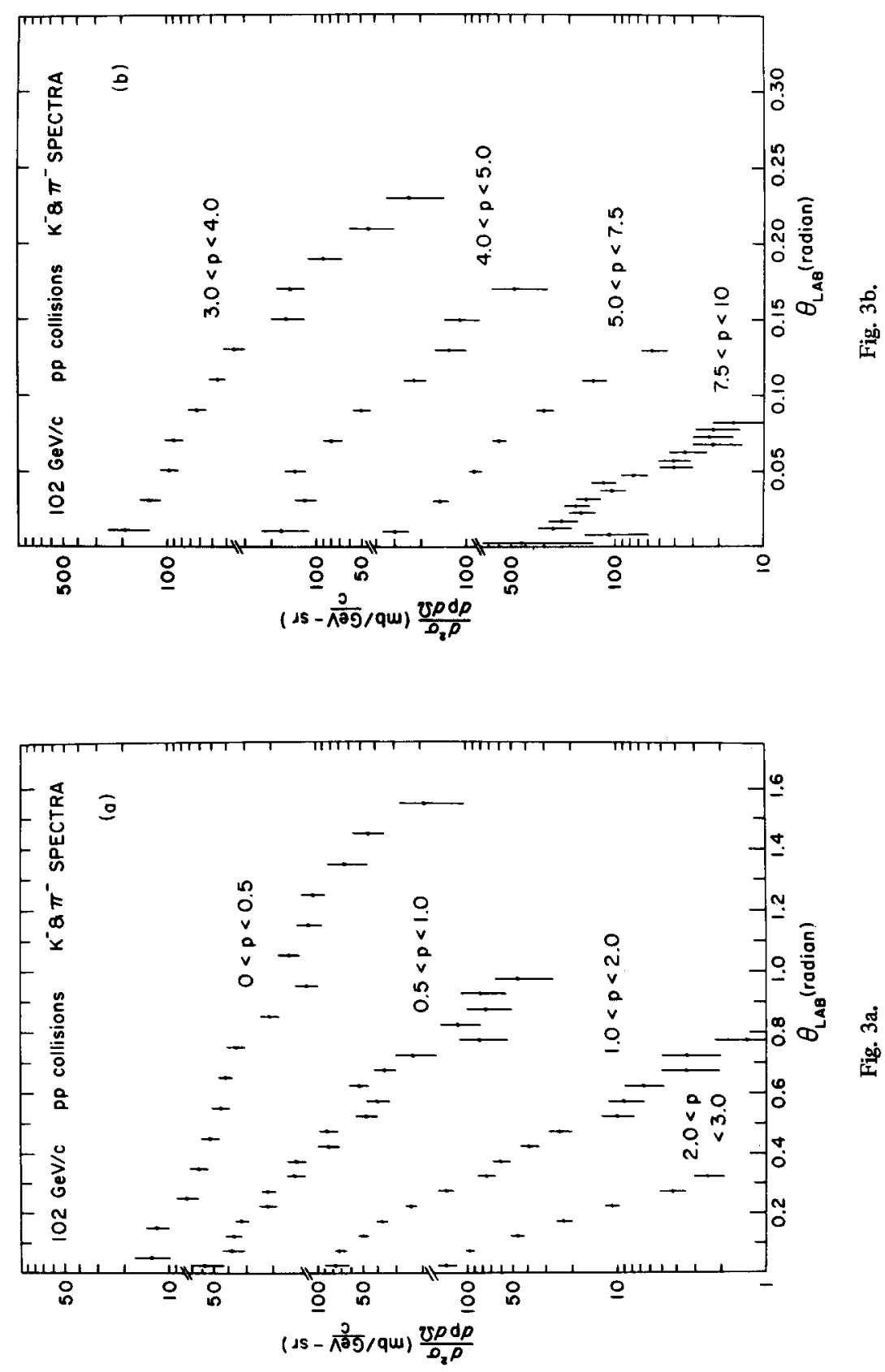


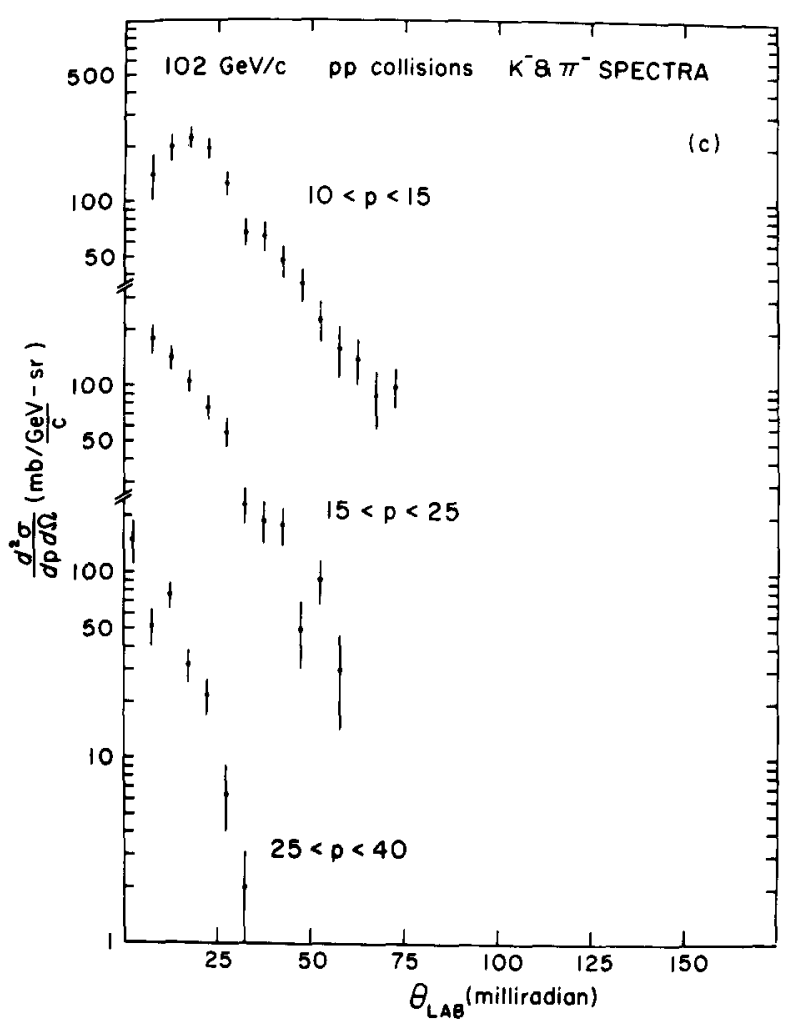

Fig. 3c.

Fig. 3. Lab angular distributions for the production in $102 \mathrm{GeV} / \mathrm{c}$ inelastic pp collisions of negatively charged pions and kaons having momenta in the indicated ranges.

in addition to statistical errors, an estimate of the systematic uncertainties in our analysis procedures.

Various subsets of the data being here prosented have been the basis for earlier publications in which we have discussed in detail many of the dynamical characteristics of proton-proton collisions at this incident energy [1-4]. In contrast, our procedure here will be to present only the experimental data without any theoretical discussion. We will individually present the data, along with the pertinent details of our analysis procedures, for the sample of secondary protons and for the charged pions and kaons.

\section{Lab angular distributions for secondary protons}

Lab angular distributions for secondary protons are presented in fig. 1. Protons 
can be reliably identified in a bubble chamber experiment by a visual estimate of ionization up to a maximum lab momentum of approximately $1.2 \mathrm{GeV} / c$. The resulting proton sample is essentially unbiased for $-1.0<x<-0.5$, where $x=P_{\ell}^{\mathrm{cm}} . / P_{\ell, \max }^{\mathrm{c} . \mathrm{m}}$ is the normalized c.m. longitudinal momentum. The overall symmetry of the proton-proton interaction can be invoked to obtain the corresponding data for positive values of $x$. In between, the proton spectra can only be estimated via an additional assumption.

Experimentally, it is found that a flat proton production spectrum $\mathrm{d} \sigma / \mathrm{d} x=$ $15 \pm 2 \mathrm{mb}$ is characteristic of high-energy proton-proton collisions for c.m. momenta in the range $-0.8<x<-0.5$. By assuming that the proton spectrum remains at this level for $|x|<0.5$, an estimate may be obtained for those protons which cannot be unambiguously identified. (We assume a transverse momentum distribution of $\mathrm{e}^{-7 P^{2}}$ for these simulated protons.) The resulting angular distributions are shown as curves in fig. 1 , rather than as data points, to emphasize that these are estimates, not measurements, and consequently should only be used with special caution.

\section{Lab angular distributions for charged pions and kaons}

The lab angular distributions for positive mesons are shown in fig. 2 , and those for negative mesons in fig. 3. Bubble chamber ionization estimates are inadequate to separate pion and kaon tracks in the momentum regions where meson production is dominant. We therefore have not attempted to separate pions and kaons in these figures; the pion mass interpretation was assumed in each case for purposes of c.m. symmetrization. Our study of $\mathrm{K}_{\mathrm{s}}^{\mathrm{o}}$ production has indicated that the kaon momentum spectrum is basically similar to that of the pions, but with a production ratio of $\left(\mathrm{K}_{\mathrm{s}}^{\mathrm{O}}\right) /\left(\pi^{-}\right)=0.06 \pm 0.01$ [4]. We have removed all identified protons from the data shown in fig. 2, and have corrected for the presence of higher momentum protons using the production model described in sect. 2; specificially, the data shown in fig. 2 have been corrected using the curves shown in fig. 1 above a lab momentum of $1 \mathrm{GeV} / c$.

We thank the members of the NAL neutrino laboratory for their aid in the taking of the exposures, and we thank our scanning and measuring personnel for their diligence.

\section{References}

[1] C. Bromberg et al., Phys. Rev. Letters 31 (1973) 1563.

[2] J. Chapman et al., Phys. Rev. Letters 32 (1974) 257.

[3] C. Bromberg et al., Phys. Rev. D, in press, report UR-459, UMBC73-19.

[4] J. Chapman et al., Phys. Letters 47B (1973) 465.

[5] M. Antinucci et al., Nuovo Cimento Letters 6 (1973) 121. 